\title{
Realidades y desafíos de la paz territorial en Colombia
}

\author{
Jerónimo Ríos \\ Universidad EAN \\ jriossie@universidadean.edu.co \\ Egoitz Gago \\ Universidad Jorge Tadeo Lozano \\ egoitz.gagoa@utadeo.edu.co
}

Recepción: 11-09-2016

Aceptación: 11-03-2017

\section{Resumen}

El objetivo principal de esta investigación pasa por responder a la siguiente pregunta: ¿cuáles son las condiciones y las posibilidades de establecer una paz territorial en Colombia? Fruto de que la dimensión territorial de la concordia se erige como uno de los conceptos más importantes del acuerdo de paz del Gobierno colombiano con las FARC y de la negociación con el ELN, de lo que se trataría sería de describir las realidades y las posibilidades de establecer una paz en clave local.

Lo anterior implica analizar las dinámicas de violencia directa y de violencia estructural que actualmente presenta el país, para lo cual se recurre a la utilización de seis regiones diferenciadas desde las que extraer conclusiones al respecto. Para ello se integran datos cuantitativos provenientes de 246 encuestas realizadas a los alcaldes y a las alcaldesas colombianos entre 2014 y 2015. De otra parte, para abordar la violencia directa, se incorporan, además, cifras facilitadas ex profeso por el Observatorio de Derechos Humanos y Derecho Internacional Humanitario adscrito a la Presidencia de la República y por la Oficina de Naciones Unidas contra la Droga y el Delito. Por su parte, la violencia estructural se analiza tomando en cuenta las cifras oficiales del Departamento de Prosperidad Social y el Departamento Nacional de Estadística.

El resultado más relevante es el de mostrar cómo en varias regiones de Colombia, especialmente en el sur y en el oriente, el problema de la violencia directa y estructural se presenta con especial notoriedad, lo cual supone uno de los principales desafíos a efectos de consolidar la paz negociada recientemente con las FARC y la que, muy posiblemente, dentro de medio plazo, tendrá lugar con el ELN.

Palabras clave: guerrillas; Colombia; violencia directa; violencia estructural; paz territorial 


\section{Abstract. Realities and challenges of territorial peace in Colombia}

The main objective of this paper is to answer the following question: What are the conditions and possibilities of territorial peace in Colombia? Given that the territorial dimension of peace constitutes one of the most important cornerstones of the Colombian government's peace agreement with the FARC and negotiations with the ELN, this paper attempts to describe the realities and challenges of local peace processes in Colombia. To achieve these aims, the dynamics of direct violence and structural violence in six regions of the country were analyzed. Quantitative data were drawn from 246 surveys of Colombian men and women mayors conducted from 2014 to 2015. To address direct violence, figures provided by the Observatory on Human Rights and International Humanitarian Law of the Office of the President and the United Nations Office on Drugs and Crime were used. Structural violence was analyzed based on official figures of the Department of Social Prosperity and the National Department of Statistics. The most relevant finding is that several regions of Colombia, especially in the south and in the east, the problem of direct and structural violence is particularly pressing. This situation is very likely to be one of the main challenges to consolidating the peace process negotiated recently with the FARC and which, quite possibly, will also take place with the ELN in the medium term.

Keywords: guerrilla; Colombia; direct violence; structural violence; peacebuilding

\section{Sumario}

1. Introducción

2. Metodología de la investigación

3. Estado de la cuestión y marco teórico

4. La violencia directa en Colombia
5. Violencia estructural e institucionalidad local en Colombia

6. Conclusiones

Referencias bibliográfica

\section{Introducción}

El siguiente trabajo tiene como propósito reflexionar sobre el alcance de uno de los términos más repetidos a lo largo de los casi cuatro años de diálogo que el Gobierno colombiano ha mantenido con las FARC: la paz territorial. Así, la paz territorial es un concepto acuñado por el comisionado de paz del equipo negociador e implica que los supuestos para la desactivación de la violencia directa y la intervención sobre los aspectos estructurales, culturales y simbólicos que tienen lugar en Colombia pasan, necesariamente, por una contextualización, una comprensión y un diseño en clave estrictamente territorial. Esto en tanto que se asume que las dinámicas locales exigen mecanismos de acción particulares y diferenciales.

Es por lo anterior que el siguiente trabajo busca indagar acerca de cuáles son esas lógicas regionales y cómo se integran con la presencia de grupos guerrilleros, como las Fuerzas Armadas Revolucionarias de Colombia (FARC) y el Ejército de Liberación Nacional (ELN). Es decir, se trataría de entender las dinámicas de violencia directa y estructural, así como su relación con el acti- 
vismo guerrillero. Todo ello en base a cifras oficiales de muy diversa índole e incorporando datos de un profundo trabajo de campo en el que se encuesta a una muestra representativa de 246 alcaldías — sobre las 1.123 que tiene el país-. Encuestas que, junto con diferentes aspectos analizados y relacionados con la violencia, darán cuenta de cómo, desde la perspectiva local, se percibe el conflicto y cuáles son las necesidades de intervención para construir una paz duradera e integral en Colombia.

Con base en lo expuesto, el trabajo se organiza en cuatro apartados. En el primero de ellos, se abordan los aspectos metodológicos a modo de pregunta de investigación, hipótesis, factores, fuentes de información y demás aspectos del diseño investigativo. En el segundo, se presenta un estado de la cuestión y un marco teórico que tienen como finalidad identificar la literatura más relevante al respecto, así como precisar y delimitar conceptualmente algunos de los elementos, enfoques y componentes teóricos más relevantes. En tercer lugar, estaría el análisis de los aspectos de la violencia más relacionados con la dimensión local. Un análisis que busca entender el conflicto en su clave regional y, particularmente, en relación con las principales dinámicas de violencia directa y de violencia estructural del mismo. Dinámicas que, en inicio, no solo deben permitir identificar las particularidades regionales, sino también el alcance y el sentido de las diferentes percepciones que las alcaldías brindan con respecto a los problemas, las demandas, las necesidades y los desafíos de una eventual paz territorial. Finalmente, en las conclusiones, además de servir de corolario a las consideraciones expuestas, se trata de plantear posibles vías expeditas que permitan continuar con una línea de investigación que debe ser trascendental en el horizonte inmediato de Colombia, frente al cual las investigaciones científicas y la universidad tendrán mucho que aportar.

\section{Metodología de la investigación}

La pregunta de partida que confiere sentido a esta investigación sería: ¿cuáles son las condiciones que permiten la materialización de la paz territorial? Inmediatamente, de esta pregunta se derivan otras posibles, como: ¿cuáles son las particularidades de la violencia directa y estructural en términos regionales?, ¿cuáles son las principales debilidades, amenazas y posibilidades percibidas desde la institucionalidad local?, ¿̨cuál es el nivel de correspondencia entre una y otra? Asimismo, todo conduce a una pregunta realizada en clave prospectiva: ¿cómo se pueden entender, dadas las circunstancias actuales, las posibilidades de abordar un proceso efectivo de posconflicto armado y construcción integral de paz?

Es decir, el punto de partida pasaría por advertir la imposibilidad de entender la construcción de paz bajo parámetros estrictamente homogéneos, topdown. Pero también de advertir las realidades locales y cómo, en según qué casos, la violencia directa y estructural afectan a la referida aspiración de una paz duradera y sostenible.

Los factores que deben permitir identificar cuál es la realidad particular que acompaña al conflicto armado son dos: la violencia directa y la violencia estruc- 
tural. La violencia directa es entendida como la violencia manifiesta, derivada de los grupos armados intervinientes en el conflicto - en este caso, las FARC y el ELN-, y la violencia estructural está relacionada con las condiciones socioeconómicas. Así, tendencias mayores de violencia directa y estructural, correspondidas con demandas desde la institucionalidad local, deberían ser elementos de necesaria atención para comprender las posibilidades reales de alcanzar una paz territorial en los términos en que se plantea el Gobierno en Colombia.

No obstante, trabajos de estas características siempre adolecen de dificultades metodológicas de diversa índole por la complejidad que supone operacionalizar un concepto tan intrincado y multifactorial como es el de violencia. Concepto que, para este caso, se reduce al número de acciones armadas unilaterales protagonizadas por las FARC y el ELN.

Una segunda complejidad, relacionada con la anterior, suele corresponderse con la fuente de información, habida cuenta de que, mayormente, los datos suelen provenir de fundaciones u observatorios de la sociedad civil. Sin embargo, en esta ocasión, los datos proceden de la fuente más confiable que existe sobre violencia directa en el conflicto colombiano: el Observatorio de Derechos Humanos y Derecho Internacional Humanitario, adscrito a la Presidencia de la República (ODHDIH). Esta entidad permitió, expresamente, el acceso a su banco de datos sobre acciones guerrilleras, las cuales se encuentran desglosadas municipalmente desde el 1 de enero de 1998 y hasta el 31 de diciembre de 2012, más los mismos datos referentes al año $2015^{1}$.

Una tercera debilidad de utilizar la violencia directa como medidor es que la dimensión latente y cultural de la misma queda desatendida, empero también es cierto que este tipo de enfoques metodológicos son los que permiten de mejor manera llevar a cabo análisis de tendencias y comprensiones de la violencia en clave regional como las que se propone este trabajo.

En lo que respectaría a la violencia estructural, esta entendida, como plantea Galtung (2003), a modo de condiciones institucionales y socioeconómicas de injusticia y de exclusión social, es operacionalizada a través de algunos indicadores de impronta socioeconómica, como el índice de calidad de vida, el coeficiente de Gini, el umbral de pobreza o los ingresos per cápita por unidad familiar. Todos ellos son obtenidos de las bases oficiales y de acceso público que publican anualmente tanto el Departamento de Prosperidad Social como el Departamento Nacional de Estadística. Sin embargo, aquí también se pueden apreciar dificultades en cuanto a su tratamiento, en la medida en que se deja de lado la dimensión política, cultural y simbólica, puesto que la violencia estructural se reduce estrictamente a aspectos socioeconómicos.

La escala geográfica de todo el análisis propuesto busca trascender más allá de divisiones estrictamente administrativas — departamentales o muni-

1. Esto es así porque los datos de 2013 y 2014, que se corresponden con los años de mayor importancia en el progreso del diálogo de paz, son confidenciales y su acceso fue denegado por parte del Ministerio de Defensa. 
cipales- De este modo, se recurre a escenarios regionales organizados por aspectos afines que permitan abordar con complejidad, pero de manera factible, un análisis profundo de la heterogeneidad que acompaña al conflicto armado colombiano a nivel local. Así, y tomando como referencia multitud de trabajos que ya han recurrido a este tipo de (re)construcciones espaciales, como Betancourt (1991), Fals Borda (1996) o el PNUD (2003), podemos decir que se diferencian, al menos, seis regiones.

Seis regiones que, ad intra, comparten similitudes en su relación con el conflicto armado, pero también respecto a sus aspectos estrictamente geográficos, culturales y socioeconómicos. Por un lado, estaría la región norte, que comprende lo que tradicionalmente se conoce como la región Caribe o de la costa atlántica, conformada por los departamentos de Córdoba, Sucre, Bolívar, La Guajira, Cesar, Atlántico y Magdalena. Se trata de una zona con una marcada idiosincrasia cultural propia y que incluso se mantiene en lo que respecta al conflicto armado colombiano, en tanto que estos siete departamentos fueron objeto de disputa y presencia de los bloques Córdoba y Norte de las AUC, del Bloque Caribe de las FARC y del Frente de Guerra Norte. En segundo lugar, estaría la región occidental, que se articula en torno al departamento de Antioquia y que sumaría los tres departamentos del Viejo Caldas - Caldas, Quindío y Risaralda - y Chocó. Esta región comportaría toda el área de influencia del Bloque Noroccidental de las FARC, el cual se extiende, stricto sensu, sobre estos cuatro departamentos, toda vez que el Frente de Guerra Occidental y el Darío Ramírez Castro, igualmente, condensaron su activismo en estos departamentos. En el oriente del país se encontrarían los departamentos de Norte de Santander, Arauca, Santander, Casanare y Boyacá, si bien, como se observará, Norte de Santander y Arauca responden a particularidades locales más similares entre sí que con respecto al resto de departamentos. Esta región se plantea como tal por ser el escenario de control del Frente de Guerra Oriental del ELN, sin duda el más poderoso de la guerrilla, y por encontrarse, igualmente, los frentes más activos de los bloques Magdalena Medio (frente 33) y Oriental (frentes 45, 10 y 28 de las FARC). En el centro del país estarían los departamentos de Cundinamarca, Meta, Tolima, Huila y Valle del Cauca, en la región que imbrica la cordillera andina con el inicio de los Llanos Orientales (Meta) y el Pacífico colombiano (Valle del Cauca). Asimismo, la región sur la conformarían los departamentos de Cauca, Nariño, Putumayo o Caquetá, por ser cuatro departamentos que comparten la zona de influencia de los frentes más activos de los bloques Sur y Occidental (frentes 6, 8, 14, 15, 29, 32, 48, 63), igualmente caracterizada por una condición selvática, fronteriza y cocalera compartida. La última región sería la amazónica y estaría integrada por Vaupés, Vichada, Guaviare, Amazonas y Guainía, que, además de presentar los menores niveles de activismo armado, conformarían la parte central de la denominada «región amazónica» en Colombia.

Violencia directa y violencia estructural, de la misma manera son analizadas no solo con base en datos e indicadores descritos, sino, igualmente, atendiendo a las percepciones locales que son recogidas y representadas a lo largo de un 
Tabla 1. Distribución por regiones de la muestra y la población municipal

\begin{tabular}{lcc}
\hline & $\begin{array}{c}\text { Número de } \\
\text { municipios encuestados }\end{array}$ & $\begin{array}{c}\text { Porcentaje de la muestra } \\
\text { sobre el total }\end{array}$ \\
\hline Región norte & 40 & $21,73 \%$ \\
Región occidental & 56 & $26,90 \%$ \\
Región central & 59 & $21,77 \%$ \\
Región oriental & 61 & $22,67 \%$ \\
Región sur & 30 & $22,20 \%$ \\
Total & $246 / 1.123$ & $21,90 \%$ \\
\hline
\end{tabular}

Fuente: elaboración propia.

trabajo de campo que, como se señalaba, incorpora 246 alcaldías encuestadas sobre un total de 1.123. Un ingente trabajo de campo que fue posible gracias al apoyo brindado por la Organización de Estados Iberoamericanos - OEI Colombia y la Federación Colombiana de Municipios (FCM)—. Ambas entidades colaboraron en el acceso a la mayoría de las alcaldías, al permitir acudir a diferentes encuentros nacionales y regionales de alcaldías colombianas, tanto en 2014 como en 2015.

Para la distribución y selección de la muestra, se hizo uso de un muestreo aleatorio simple, de manera que la distribución de alcaldías encuestadas fue la siguiente: 40 municipios de la región norte, 56 municipios de la región occidental, 59 municipios de la región central, 61 municipios de la región oriental y, finalmente, 30 municipios de la región $\operatorname{sur}^{2}$.

De acuerdo con estos datos, el volumen de la muestra sería considerable, si bien, en términos de rigor metodológico, el ideal para unos parámetros de análisis sobre un nivel de confianza del 95\% y un error en la estimación muestral del 5\% hubiera sido de 286 alcaldías. Es decir, cuarenta alcaldías más de las finalmente trabajadas, lo cual, si bien permite mantener niveles de confianza del 95\%, engrosa en medio punto la posible desviación en el error muestral. No obstante, esta vicisitud no debiera restar valor al trabajo de campo realizado, en buena medida porque la complejidad del objeto de estudio, la dificultad en el acceso a fuentes, la renuencia, en muchas ocasiones, a participar en trabajos de este tipo, así como problemas de accesibilidad, disponibilidad o el propio hecho del fin del mandato municipal (para noviembre de 2015) hicieron que, finalmente, por razones de tiempo y de efectividad, se decidiese acotar la investigación con el total de cuestionarios disponibles.

En lo que respectaría a la metodología de la encuesta, conviene precisar que todas las encuestas fueron realizadas a alcaldes y a alcaldesas del país, a quienes se garantizó en todo momento la confidencialidad y el anonimato de

2. Dados los problemas de accesibilidad, de la región amazónica, conformada por Vaupés (3 municipios), Vichada (4 municipios), Amazonas (2 municipios) y Guainía (1 municipio), solo se consiguió encuestar a un alcalde. Sea como fuere, tanto el número de municipios como la presencia marginal de grupos armados hizo que, finalmente, se desestimase su análisis. 
Tabla 2. Medición de la confiabilidad de la muestra de alcaldes objeto de estudio ( $N$ tamaño del universo): $1.123 ; p$ (probabilidad de ocurrencia): 0,5

\begin{tabular}{|c|c|c|c|c|c|c|c|c|c|c|}
\hline \multicolumn{2}{|c|}{$\begin{array}{l}\text { Nivel de confianza } \\
\text { (alfa) }\end{array}$} & 1-alfa/2 & \multicolumn{2}{|c|}{ Z (1-alfa/2) } & \multicolumn{6}{|c|}{ Fórmula empleada } \\
\hline $90 \%$ & & 0,050 & & & \multirow{4}{*}{\multicolumn{6}{|c|}{$\frac{N=n_{0}}{1+n_{0} / n}$ donde: $n_{0}=p^{*}(1-p) *[z(1-\alpha / 2) / d]^{2}$}} \\
\hline $95 \%$ & & 0,025 & & & & & & & & \\
\hline $97 \%$ & & 0,015 & & & & & & & & \\
\hline $99 \%$ & & 0,005 & & & & & & & & \\
\hline \multicolumn{11}{|c|}{ Matriz de tamaños muestrales para un universo de 1,123 con una $p$ de 0,5} \\
\hline \multirow[t]{2}{*}{$\begin{array}{l}\text { Nivel de } \\
\text { confianza }\end{array}$} & \multicolumn{10}{|c|}{$d$ (error máximo de estimación) } \\
\hline & $10,0 \%$ & $9,0 \%$ & $8,0 \%$ & $7,0 \%$ & $6,0 \%$ & $5,0 \%$ & $4,0 \%$ & $3,0 \%$ & $2,0 \%$ & $1,0 \%$ \\
\hline $90 \%$ & 63 & 77 & 96 & 122 & 160 & 217 & 306 & 449 & 673 & 962 \\
\hline $95 \%$ & 88 & 107 & 132 & 167 & 216 & 286 & 391 & 547 & 765 & 1005 \\
\hline $97 \%$ & 107 & 129 & 158 & 198 & 253 & 332 & 445 & 604 & 813 & 1025 \\
\hline $99 \%$ & 145 & 174 & 211 & 261 & 327 & 418 & 540 & 699 & 884 & 1052 \\
\hline
\end{tabular}

Fuente: elaboración propia.

las respuestas. Es por ello que los desgloses son estrictamente departamentales o regionales, sin entrar en los datos específicos en referencia a municipios. El total de preguntas fueron catorce y, en todo momento, el cuestionario fue socializado previamente a su aplicación con la propia FCM, a efectos de evitar problemas de comprensión o de contenido y optimizar los niveles de adecuación en la respuesta.

\section{Estado de la cuestión y marco teórico}

Los estudios sobre el conflicto colombiano aglutinan un ingente volumen de trabajos académicos de diferente enfoque, objeto e interpretación. Es decir, abunda una prolija literatura sobre la historia del conflicto armado, ya sea desde sus orígenes (Fals Borda et al., 1962; Oquist, 1978; Sánchez y Meertens, 1983) o bien desde el devenir de los diferentes actores involucrados. Hay trabajos que se centran en las guerrillas de las FARC (Pécaut, 2008; Aguilera, 2010); en el ELN (Medina, 1996, 2011); en el EPL (Calvo, 1986), o en el M-19 (Lara, 2014).

Un segundo gran bloque de trabajos se concentra en otros actores concomitantes, ya sean los cárteles o el paramilitarismo (Medina, 1990; Ronderos, 2014). Y otros muchos aportes ponen su atención en las fuentes de financiación de los diferentes grupos armados, tanto de las guerrillas (Bejarano y Pizarro, 2001) como de los paramilitares (Reyes, 2009; Romero, 2011), siendo especialmente preponderante la atención por el impacto del narcotráfico.

Una tercera vía de trabajos centraría su atención en las políticas de seguridad llevadas a cabo en la gestión del conflicto armado interno. Existen investigaciones que analizan las políticas de seguridad nacional (Blair, 1993; Leal, 
1994), mientras que otras atienden a las políticas de cooperación internacional, especialmente el Plan Colombia (Otero, 2010; Rojas, 2015) y los Laboratorios de Paz de la Unión Europea (Barreto, 2016).

Finalmente, habría trabajos en perspectiva regional que tienen como principal intención explicar las relaciones entre violencia y territorio, bien con base en recursos económicos y control de enclaves estratégicos (PNUD, 2003; Echandía, 2006; Salas, 2015), bien tratando de profundizar en dinámicas de estricto carácter regional (Torres, 2011; Vásquez et al., 2011).

Sin embargo, y como se puede observar, la línea sobre trabajos de posconflicto armado es relativamente nueva y, por su actualidad, ofrece posibilidades para realizar análisis de todo tipo, si bien hasta el momento predominan los esfuerzos mayormente generalistas y de escasa profundidad académica. Trabajos recientes, como el de Morales (2015) o Ávila y Valencia (2016), incurren más en elementos normativos y deontológicos de la construcción de paz, mientras que otros, como los de López de la Roche (2015) o Cárdenas (2015), se encuentran más próximos a los análisis del discurso. Expresado de otro modo, hasta el momento, los trabajos de investigación de mayor profundidad y rigor científico-social estarían por llegar.

Sea como fuere, cualquier intento de investigar un conflicto armado como el colombiano, especialmente en lo que respecta a la investigación para la paz, debe tener, como punto de partida, de algún modo, una definición de paz que, ni mucho menos, resulta sencilla de acotar.

$\mathrm{Al}$ respecto, y a grandes rasgos, siguiendo la clasificación que plantea Harto de Vera (2004: 164-198), cabe identificar, al menos, cuatro grandes clasificaciones que ordenan los enfoques que dominan la investigación para la paz. En el primero de los planteamientos, Curle (1976) o Eide (1974) distinguen entre corrientes minimalistas, intermedias y maximalistas. Serían corrientes minimalistas aquellas que entienden la paz como la ausencia de guerra, especialmente en la esfera internacional. Las corrientes intermedias irían más allá, al entender la paz tanto como la ausencia de guerra como la ausencia de amenazas a la misma, mientras que el enfoque maximalista abogaría por la comprensión de la paz como la ausencia de violencia directa, estructural y cultural, bajo una óptica tanto nacional como internacional. Este enfoque maximalista, además, comprende la paz como un instrumento.

Un segundo enfoque, planteado por Boulding (1977), propondría un planteamiento igualmente tridimensional, pero dual a su vez, en cada una de sus categorías. El propio Boulding, desde la negación de la violencia como instrumento, considera que las estructuras son resultados naturales de un proceso constante de interacción y de evolución que, según su interpretación, pueden ser objeto de «relativa predicción». De ahí su autoposición como evolucionista, aunque esta posición permita opciones, igualmente, que no rechazan la violencia, como las invocadas por Hardin (1983). En un segundo grupo estarían los planteamientos que interpretan las estructuras en términos dialécticos, propiamente marxistas, ya sean en clave nacional, cultural o económica, y que, por extensión, como factor de cambio, casi siempre acogen de buen grado el 
recurso de la violencia como factor de transformación. Quizá teóricos como Schmid (1968) representarían a la escasa excepción dentro de este enfoque. Por último, desde el estructuralismo "más ortodoxo", se entendería que la estructura, a pesar de todo, termina siendo estática espacio-temporalmente. Esto es, de acuerdo con autores como Rummel (1998) o Galtung (2003), quienes coinciden, especialmente, en su rechazo a la violencia como medio para obtener la paz. Frente a ellos se encontrarían referentes weberianos o elitistas, que no terminarían por rechazar el empleo de la violencia como medio de transformación.

Un tercer intento de clasificación de las diferentes teorías sobre la resolución de conflictos sería el planteado por Röling (1984) y Dedring (1981), quienes basan su conceptualización en función de los métodos de análisis empleados, distinguiendo entre cualitativos y cuantitativos. Esto se entiende, en buena medida, por la marcada influencia de la ciencia política estadounidense, desde la cual los trabajos cuantitativos, con dosis de tratamiento estadístico y matemático, serían denominados «enfoques duros», frente a los estudios simbólicos o discursivos sobre la violencia o la paz, que, con técnicas de investigación cualitativa, serían categorizados como «enfoques blandos».

Por último, estarían los enfoques planteados por Miall et al. (2015), que, como en los casos anteriores, responden a un intento de clasificación tripartita. En primer lugar, se encontraría la corriente de tipo realista, basada en términos estrictamente de paz negativa y modelos cuantitativos y que tiene como referencia las aportaciones de Rapoport (1960) y Boulding (1962). En un segundo enfoque se encontraría el planteamiento estructuralista, impulsado por Galtung desde la década de 1960 y que, sumando la noción de paz positiva, aboga por el recurso a métodos cualitativos de análisis. Finalmente, el enfoque pluralista, planteado por Azar (1990), trataría de integrar ambas posiciones bajo la noción de "conflicto social prolongado». Esto es, un intento de señalar que, en muchos casos, los conflictos son multidimensionales en sus factores, desarrollos y actores, de modo que el origen y la forma de resolución terminan por converger en una frontera difusa e imprecisa que impide utilizar parámetros estancos de análisis o intervención.

Es con base a lo expuesto que este trabajo se aproximaría más a la dimensión maximalista y estructuralista, que concibe la paz no solo como la ausencia de guerra, tal y como la perciben los enfoques minimalistas y más conservadores, sino como la ausencia de las condiciones estructurales (socioeconómicas) y culturales (símbolos y valores) que sostienen y retroalimentan la violencia. Dicho de otro modo, se trataría de entender la paz como la ausencia tanto de violencia directa como estructural o cultural, si bien, particularmente, el trabajo se concentra en las dos primeras dimensiones y descarta la tercera por limitaciones estrictamente metodológicas.

Más allá de la clásica noción de paz positiva, resulta imprescindible conferir a la misma mayores visos de realidad, tal y como sugiere Muñoz (2000), es decir, entendiendo la paz como un escenario dinámico, en continua construcción, y no solo en términos ideales de Galtung. De lo que se trata es de des- 
mitificar la paz y, por extensión, de abandonar las ideas de perfección, utopía, lejanía y totalidad que acompañan a la paz, toda vez que la imposibilitan. Todo lo contrario, la paz positiva debe entenderse en términos realistas, como un proceso inacabado, sustantivado por continuas dialécticas tanto posibilistas como reformistas, pragmáticas y transformadoras. Dialécticas que son particulares en cada situación local, habida cuenta de que las expresiones de la violencia directa y estructural asumen diferentes causas y representaciones. Es por ello que la noción de paz territorial a la que alude el Gobierno colombiano, en la teoría, se ajustaría perfectamente a la intrincada y heterogénea realidad que caracteriza al conflicto armado interno.

Así, hablar de paz territorial supondría reconocer que la paz debe materializarse sobre la base de un enfoque de derechos con perspectiva territorial. Igualmente, implica reconocer las realidades de exclusión social, marginalidad, falta de oportunidades y debilidad institucional que impera en buena parte del país y que son un sustento sobre el que, necesariamente, se debe intervenir si de desactivar el conflicto armado se trata.

Quizá es por ello que, desde hace meses, el mismo gobierno viene emplazando a un proceso de construcción de paz bajo dinámicas participativas y de enfoque diferencial territorial, desde las cuales se puedan construir acciones que permitan, regionalmente, recomponer el país a través del proceso de paz. Una cuestión que engarza con la paz positiva, pero que requiere comprender cómo y cuáles son las dinámicas de la violencia a nivel local. Se trata de operar en términos de realidad y deseabilidad, de manera que un buen análisis de los niveles de violencia directa y estructural, unido a una comprensión de los retos y de los desafíos de la institucionalidad local, deben aportar a la factibilidad de un proceso integral de construcción de paz.

\section{La violencia directa en Colombia}

El conflicto colombiano, aunque tiene su origen formal a mediados de los años sesenta, cuando aparecen las estructuras guerrilleras como las FARC y el ELN, lo cierto es que hunde sus raíces en los años cuarenta e, incluso, en los años treinta, por ser cuando aparecen las primeras guerrillas campesinas, inspiradas por las luchas agrarias del momento (Gilhodès, 1972). Lo cierto es que este conflicto es el más longevo y violento de todo el hemisferio occidental, al dejar consigo más de 200.000 víctimas mortales. Incluso en algún momento, especialmente a inicios de la década pasada, tales fueron los niveles de violencia exhibidos, que Colombia pasó a consolidarse como el «Estado fallido» latinoamericano por antonomasia (Rotberg, 2004).

La verdad es que, para aquel entonces, tales consideraciones no resultaron cuestión baladí. Una guerrilla como las FARC superaba los 70 frentes de guerra y controlaba más de 350 municipios del país, gracias a los cerca de 18.000 efectivos, 60.000 colaboradores y unos ingresos que superaban los 1.500 millones de dólares anuales. Además, el ELN, la segunda guerrilla del país, a finales de los años noventa, llegaba casi a los 5.000 combatientes y ejer- 
cía el control efectivo sobre unos 150 municipios. Por si fuera poco, en más de 200 municipios, casi todos en el norte del país, se habían consolidado los grupos paramilitares - desde septiembre de 1997, organizados en torno a las siglas AUC (Autodefensas Unidas de Colombia) — e, igualmente, aglutinaban a más de 12.000 integrantes (Ríos, 2017).

De hecho, bajo esta coyuntura, solo entre 1998 y 2002, se produjeron en Colombia 17.818 infracciones al Derecho Internacional Humanitario y 17.043 violaciones a los derechos humanos. La violencia política y social se cobró 18.595 víctimas en estos cinco años y las muertes producidas por acciones bélicas ascendieron a 14.342 (Otero, 2007: 14). Es más, el número de desplazados llegó a los mayores niveles de la historia de Colombia, pues solo entre 1998 y 2002 se produjeron, según CODHES (2011: 18), 729.928 casos de desplazamiento forzado. Una tesitura que, sin embargo, permitirá entender cómo y bajo qué circunstancias se produce la llegada de Álvaro Uribe a la presidencia del país y cómo bajo su mandato tienen lugar buena parte de las transformaciones que caracterizarán a las dinámicas de la violencia derivada del conflicto en el transcurso de la última década.

Una vez que llega Álvaro Uribe al poder, se inicia en Colombia toda una política de seguridad que tiene como leitmotiv la confrontación armada y el debilitamiento de las guerrillas como nunca antes se había llevado a cabo. Durante ocho años consecutivos, se destinó un 5\% del PIB a seguridad y defensa, a lo que se sumaron otros 8.000 millones de dólares provenientes de la cooperación estadounidense a través del Plan Colombia. Entre otras cosas, ello permitió que, entre 2002 y 2010, la Policía Nacional colombiana pasara de los 110.000 miembros a los 160.000 y que las fuerzas militares se incrementaran de 203.000 a 270.000 integrantes. A su vez, se experimentaron otros tantos avances notables en lo que tuvo que ver con modernización, organización, coordinación, distribución y disposición de recursos (Ministerio de Defensa, 2010: 68).

Transcurridos estos ocho años, entre 2002 y 2010 , y resultado de todo lo anterior, se destruyeron 20.062 laboratorios cocaleros y se incautaron 1.233 toneladas de marihuana, 5,3 toneladas de heroína y 1.269 toneladas de cocaína. Si en 2002 la superficie de coca era de 130.364 ha, en 2010 la superficie había disminuido a 58.073 ha (UNODC, 2013). De igual manera, las FARC redujeron su pie de fuerza a poco más de 8.000 efectivos con presencia en 166 municipios, mientras que el ELN veía mermado el número de efectivos a menos de 2.000 sobre apenas 27 municipios (ODHDIH, s. f.).

La gran mayoría de todo este conjunto de acciones y cifras condujeron a que algunos estudios estimasen que, entre 2002 y 2010 , el ELN perdió hasta un $77 \%$ de su fuerza de combate, por un $83 \%$ para el caso de las FARC (Fundación Seguridad y Democracia, 2008: 5). Todo gracias a un esquema de intervención sobre el aspecto de seguridad que se consolidó con la intención de fortalecer la estructura de la inteligencia en seguridad, identificando con claridad las funciones y el alcance de cada uno de los grupos de la fuerza pública. También se priorizó el fortalecimiento del Estado sobre ciertas regiones, 
especializando los escuadrones y las unidades de combate y ganando presencia, fundamentalmente, en enclaves rurales, tradicionalmente de especial arraigo guerrillero.

En el fondo, esta aparente victoria de la política de seguridad democrática resultó ser una forma minimalista, cuantitativa y conservadora de reducir la noción de paz a la mera ausencia de guerra, pero lo que más bien propició fue, sí, debilitar a las estructuras guerrilleras de las FARC y el ELN, y, a la vez, regionalizar el conflicto armado y enquistarlo allí donde concurrían una serie de condiciones favorables para su supervivencia y continuismo, lo cual, por ejemplo, se puede apreciar mayormente en el modo de presentarse el conflicto armado colombiano en los departamentos del sur del país o del nororiente, como Arauca y Norte de Santander ${ }^{3}$.

Si se observa la tabla 3, se puede apreciar una particular regionalización del conflicto armado que pone de manifiesto, por ejemplo, cómo los departamentos del sur del país (por ejemplo: Caquetá, Cauca, Nariño y Putumayo) han concentrado más de la mitad de las acciones armadas de las FARC entre 2010 y 2012. Asimismo, permite observar de qué modo el ELN concentraría hasta un $55 \%$ de sus acciones guerrilleras en la región oriental y, concretamente, en los departamentos de Arauca y Norte de Santander.

Dicho de otro modo, algunos de los departamentos más alejados del centro del país, como los ya mencionados de Cauca, Caquetá, Nariño, Putumayo, Arauca y Norte de Santander, entre 2010 y 2012, se erigieron como los que mostraban mayor activismo armado por parte de las guerrillas ${ }^{4}$. Activismo igualmente relacionado con la actividad cocalera, pues, con la excepción de Arauca, tal y como informa UNODC (2013), estos serían los departamentos, por antonomasia, más cocaleros del país.

Sin embargo, en los últimos años, las tendencias de regionalización y narcotización de esta violencia guerrillera apenas han podido ser estudiadas, pues el gobierno ha preferido ser cauto al respecto y no hacer públicos los datos sobre violencia guerrillera u operativos de la Fuerza Pública en 2013 y 2014. No obstante, esta ausencia de datos se podría mitigar, a efectos de este trabajo, de dos maneras: atendiendo a las percepciones que las alcaldías reconocen con

3. A pesar de que metodológicamente se utilizan escalas regionales para analizar y presentar la evolución del conflicto armado, en las diferentes tablas se presentan Arauca y Norte de Santander aún integrados en la región oriental de manera particular. Esto a efectos de mostrar que, a pesar de que la región oriental la conformarían los departamentos de Santander, Casanare, Boyacá, Arauca y Norte de Santander, prácticamente son estos dos los que concentran los peores niveles de manera muy significativa, tanto respecto a violencia directa como a violencia estructural.

4. Entre 2010 y 2012 se registraron en Colombia 2.414 acciones guerrilleras. Los departamentos con mayor activismo armado fueron Cauca (490), Nariño (299), Antioquia (250), Arauca (204), Norte de Santander (198), Putumayo (172), Meta (150) y Caquetá (149). De estos ocho departamentos, con la excepción de Antioquia (región occidental) y Meta (región central), se puede observar un relativo enquistamiento en el sur y el nororiente de Colombia, al tratarse de departamentos de índole fronteriza y alejada de los centros decisorios del país (ODHDIH, s. f.). 
Tabla 3. Periferialización del conflicto armado, 2010-2012

\begin{tabular}{lrrrr}
\hline \multicolumn{1}{c}{ Período 2010-2012 } & ELN & FARC & Fuerza pública & Hectáreas de coca \\
\hline Región occidental & 22 & 286 & 354 & 20.385 \\
Región norte & 3 & 79 & 194 & 13.899 \\
Región central & 0 & 349 & 1.021 & 10.925 \\
Región oriental $^{\star}$ & $110(103)$ & $319(299)$ & $366(315)$ & 11.943 \\
Región sur $_{\text {Región amazónica }}$ & 52 & 1.068 & 1.080 & 90.699 \\
Total & 0 & 37 & 185 & 27.513 \\
\hline
\end{tabular}

* Entre paréntesis, los datos para Arauca y Norte de Santander.

Fuente: elaboración propia con base en los históricos de UNODC y ODHDIH.

respecto a la presencia de grupos armados, en tanto que fueron encuestados en 2014 y 2015, y analizando los datos que el Ministerio de Defensa ha hecho públicos, exclusivamente, para el año 2015.

Lo que cabría advertir, con base en estas dos fuentes de información, es que la regionalización y la narcotización, lejos de mantenerse estables, se han agudizado muy sustancialmente en los últimos años. Por ejemplo, cuando se preguntaba a los alcaldes y a las alcaldesas sobre las percepciones de presencia de grupos armados y la evolución en el tiempo, se puede observar cómo en las regiones sur y oriental se presentan, particularmente, los peores registros, tal y como recoge la tabla 4.

Se evidencia una correspondencia de las percepciones, tanto con los indicadores de violencia existentes como con la lógica de los trabajos de cariz regional que, durante las últimas décadas, han investigado el conflicto armado. Así, hacia 1998, como sería de esperar, se percibe mayor presencia paramilitar en los departamentos de la región atlántica, que es donde mayor arraigo y alcance tuvo el proyecto de las AUC. Asimismo, hacia 1998, las FARC presentan mayores percepciones de presencia en el sur, mientras que el ELN lo hace en el nororiente. Sin embargo, cuando se trata de observar la percepción de presencia de grupos armados en 2014 y 2015, lo verdaderamente sorprendente es observar cómo los cuestionarios arrojan tendencias de continuidad, prácticamente calcadas en términos regionales, respecto de los diferentes grupos armados. Esto

Tabla 4. Percepción de los alcaldes y de las alcaldesas sobre la presencia de FARC, ELN y paramilitares, en 1998 y en 2014

\begin{tabular}{lcccccc}
\hline & AUC (1998) & FARC (1998) & ELN (1998) & Bacrim (2104) & FARC (2014) & ELN (2014) \\
\hline Región occidental & 3,30 & 4,55 & 2,63 & 2,47 & 2,67 & 1,94 \\
Región norte & 6,16 & 3,25 & 2,91 & 6,01 & 1,87 & 1,37 \\
Región centro & 4,04 & 4,09 & 1,83 & 2,86 & 3,65 & 1,14 \\
Región oriental & 3,89 & 4,78 & 6,01 & 2,51 & 4,01 & 4,18 \\
Región sur & 4,70 & 8,01 & 2,70 & 3,85 & 8,10 & 2,28 \\
\hline
\end{tabular}

Fuente: elaboración propia. 
se debe a que las Bacrim — grupos herederos de los paramilitares— continúan siendo percibidos con mayor notoriedad en el norte, mientras que las FARC lo hacen en el sur y el ELN, en el nororiente. Así, aunque la mayor presencia de activismo guerrillero en las regiones sur y oriental pudiera entenderse como algo que siempre estuvo, en la medida en que los corredores periféricos tuvieron una ingente presencia de grupos armados irregulares, esta pareciera que se ha intensificado, al intuir que, tras la PSD y las nuevas dinámicas de la violencia, la presencia de grupos se hubiera enquistado en aquellos enclaves donde las ventajas locales y el arraigo regional siempre resultaron favorables a las guerrillas de las FARC y del ELN. También porque, en estas regiones, la presencia del paramilitarismo de las AUC fue estrictamente residual, y cuando apareció, con la intención de disputar a la guerrilla el control de los recursos provenientes del narcotráfico, nunca se trató de un actor con posibilidades de obtener ninguna victoria militar sobre la guerrilla. Cuestión bien diferente, por ejemplo, con respecto a la región norte, que fue su escenario de mayor arraigo y desarrollo, por la presencia hegemónica sobre todo del Bloque Norte y del Bloque Córdoba, lo que explicaría, además de la menor presencia guerrillera, la mayor notoriedad de grupos herederos del paramilitarismo.

De igual forma, si se observan los únicos datos disponibles para 2015, las conclusiones que se pueden extraer se integran igualmente en lo anterior. En primer lugar, cabe apreciar una importante concentración de las actividades guerrilleras en las regiones sur y oriental, lo cual se pone de manifiesto en el hecho de que, de las 122 acciones guerrilleras reportadas por el Ministerio de Defensa, 44 se concentrarían en Cauca, Nariño, Caquetá y Putumayo, y otras 38 en los departamentos fronterizos de la región oriental en Arauca y Norte de Santander. De igual manera, y con base en el desglose departamental de municipios cocaleros en Colombia reportado por UNODC en el año 2015,

Gráfico 1. Porcentaje de municipios y de acciones guerrilleras coincidentes con cultivos cocaleros, 2001-2015

100

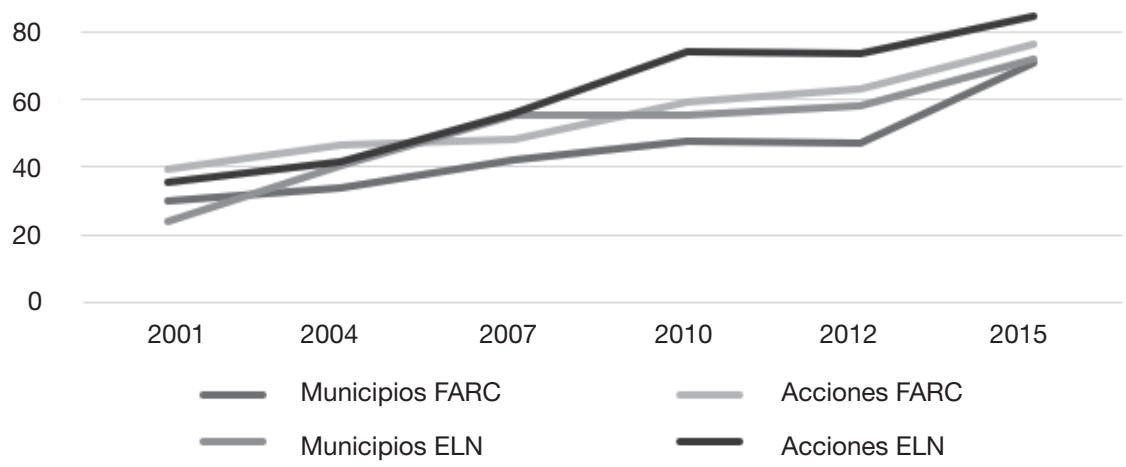

Fuente: elaboración propia. 
Tabla 5. Evolución de las acciones guerrilleras y de los cultivos de coca, 2012-2015

\begin{tabular}{|c|c|c|c|c|c|}
\hline & $\begin{array}{c}\text { Acciones } \\
\text { guerrilleras } \\
\text { en } 2012\end{array}$ & $\begin{array}{l}\text { Acciones } \\
\text { guerrilleras } \\
\text { en } 2015\end{array}$ & $\begin{array}{l}\text { Cultivos } \\
\text { de coca } \\
\text { en } 2012\end{array}$ & $\begin{array}{l}\text { Cultivos } \\
\text { de coca } \\
\text { en } 2015\end{array}$ & $\begin{array}{c}\% \text { variación } \\
\text { cultivos } \\
2012-2015\end{array}$ \\
\hline Región occidental & 122 & 22 & 6.170 ha & 3.891 ha & $-36,9 \%$ \\
\hline Región centro & 160 & 6 & 3.181 ha & 5.692 ha & $+78,9 \%$ \\
\hline Región norte & 37 & 6 & $3.061 \mathrm{ha}$ & 2.446 ha & $-20,0 \%$ \\
\hline Región sur & 381 & 44 & 24.901 ha & 66.195 ha & $+165,8 \%$ \\
\hline Región oriental & 179 & 39 & 4.527 ha & $11.573 \mathrm{ha}$ & $+155,6 \%$ \\
\hline \multirow[t]{2}{*}{ Región amazónica } & 16 & 4 & 5.746 ha & 6.287 ha & $+9,2 \%$ \\
\hline & 895 & 122 & 47.790 ha & 96.084 ha & $+101,0 \%$ \\
\hline
\end{tabular}

Fuente: elaboración propia con base en los registros del Ministerio de Defensa y de UNODC.

de los 70 municipios donde se registró presencia guerrillera, en 54 de ellos concurrieron cultivos ilícitos, lo cual representa más de un 77\%. Por último, de las mismas 122 acciones guerrilleras, 97 habrían sucedido en contextos con presencia de cultivo ilícito, lo cual representaría un volumen de casi el 80\%. Ello implicaría que las dinámicas ya advertidas en 2012 se habrían agravado durante los tres años que duraron las negociaciones de La Habana, tal y como puede observarse en el gráfico 1 .

De lo anterior, igualmente se deduciría una consideración que invita al optimismo, pues la intensidad del conflicto se ha reducido muy sustancialmente. A su vez, se pone de manifiesto cómo para 2015, en el oriente colombiano, se registró actividad guerrillera de las FARC y del ELN en todos los municipios de Arauca y en toda la región del Catatumbo de Norte de Santander. Igualmente, en el sur del país, las FARC siguen presentando ingentes niveles de activismo y se aprecian posibles lógicas de continuidad con el crimen organizado, por tratarse, ambas regiones, de las más cocaleras del país.

\section{Violencia estructural e institucionalidad local en Colombia}

Además de lo expuesto hasta el momento, son muchos los trabajos que, a efectos de comprender las lógicas del conflicto armado colombiano, han reivindicado la importancia tanto de la saqueabilidad de sus recursos - especialmente el control sobre la tierra (Reyes, 2009), el narcotráfico (Ríos, 2016) y la minería ilegal (Massé y Camargo, 2012), entre otros-, como, en términos más amplios, del factor de la violencia estructural. Desde el trabajo de Sánchez (1987), esta violencia estructural ha marcado una férrea línea de interpretación, especialmente y sobre todo a partir de los años noventa, con trabajos como los de Reyes (1988) o Ramírez (1990), y que fue desarrollada durante toda la década pasada por el Instituto de Estudios Políticos y Relaciones Internacionales de la Universidad Nacional de Colombia.

Y es que, como se puede ver en la tabla 6, cuando se pregunta a las alcaldías sobre la valoración de los principales problemas del municipio, cabe extraer diferentes consideraciones. En primer lugar, destaca la notable diferencia que 
Tabla 6. Valoración de las alcaldías sobre los problemas del municipio, 2014-2015

\begin{tabular}{lccccccc}
\hline & Desempleo & Pobreza & Inequidad & $\begin{array}{c}\text { Ausencia } \\
\text { del Estado }\end{array}$ & $\begin{array}{c}\text { Presencia } \\
\text { de grupos }\end{array}$ & Corrupción & $\begin{array}{c}\text { Cultivos } \\
\text { ilícitos }\end{array}$ \\
\hline Norte & 5,92 & 5,70 & 5,13 & 4,58 & 4,69 & 6,26 & 3,06 \\
Occidente & 7,14 & 5,69 & 5,61 & 4,30 & 3,91 & 3,60 & 2,90 \\
Central & 6,98 & 6,04 & 4,63 & 4,32 & 4,19 & 3,06 & 2,63 \\
Oriente $^{*}$ & 5,22 & 5,85 & 5,61 & 6,10 & 5,74 & 2,68 & 2,71 \\
& $(7,00)$ & $(6,14)$ & $(6,01)$ & $(7,55)$ & $(8,65)$ & $(3,0)$ & $(5,0)$ \\
Sur & 7,93 & 8,14 & 6,43 & 7,57 & 8,15 & 4,00 & 7,14 \\
Media nacional & 6,53 & 6,50 & 5,81 & 5,37 & 5,04 & 4,01 & 3,74 \\
\hline
\end{tabular}

* Entre paréntesis, los datos para Arauca y Norte de Santander.

Fuente: elaboración propia.

hay entre las valoraciones que recibe la violencia estructural frente a la violencia directa. Es decir, de los siete problemas que se plantean a las alcaldías (desempleo, pobreza, inequidad, ausencia del Estado ${ }^{5}$, presencia de grupos armados, corrupción y concurrencia de cultivos ilícitos), es destacable que los de mayor consideración, los cuatro primeros, corresponden a factores estrictamente estructurales.

En la mayoría de los municipios encuestados, el desempleo, la pobreza, la inequidad y el abandono del Estado fungen como las cuatro principales preocupaciones, con unos registros, como se puede observar, muy superiores a los factores más relacionados con la violencia directa, como son la presencia de grupos o la misma concurrencia de cultivos. Esta circunstancia permitiría entender que, si bien la violencia estructural presenta niveles tan notables como ubicuos en buena parte del país, la violencia directa derivada del conflicto incorpora una mayor focalización territorial.

Si se observa con detalle, es en los departamentos del sur y en el nororiente donde el problema representado por la presencia de grupos muestra la mayor preocupación para la institucionalidad local. Igualmente, el problema del cultivo ilícito es mucho más relevante en estos dos enclaves, tanto que mientras que en el país la preocupación por la concurrencia de cultivos ilícitos tiene un valor promedio entre los alcaldes encuestados de 3,74, esta casi se duplica cuando se trata de la región sur $(7,14)$ o Norte de Santander, donde el registro supera los 7 puntos de valoración entre las alcaldías encuestadas.

Como también se puede observar, las consideraciones expuestas se corresponderían perfectamente con los datos facilitados por las entidades consultadas, no solo en relación con la violencia directa, sino, igualmente, con la violencia estructural recogida en la tabla 7 . Y es que la fractura entre el centro y la periferia también se pondría de manifiesto de manera considerable en los indicadores y en las cifras macroeconómicas que presenta el país. En términos, por ejemplo,

5. Entendida la ausencia del Estado en términos de inversión, infraestructura o arquitectura institucional. 
Tabla 7. Indicadores de violencia estructural, 2014

\begin{tabular}{lrrrr}
\hline & PIB p. c. & Gini & Línea de pobreza & \multicolumn{1}{c}{ ICV } \\
\hline Norte & 341.409 & 0,500 & 46,3 & 63,60 \\
Occidente & 454.116 & 0,532 & 36,8 & 41,41 \\
Central & 551.555 & 0,499 & 22,8 & 40,09 \\
Oriente* $^{*}$ & 419.855 & 0,513 & 41,4 & 54,97 \\
Sur & & $(0,519)$ & $(44,70)$ & $(61,58)$ \\
Amazonas & 289.945 & 0,514 & 49,5 & 56,67 \\
Media nacional & - & - & - & - \\
\hline
\end{tabular}

* Entre paréntesis, los datos para Arauca y Norte de Santander.

** Sin datos.

Fuente: elaboración propia con base en los datos del DANE y el DPS.

de PIB per cápita por unidad de gasto familiar ${ }^{6}$, las regiones central y occidental, y especialmente departamentos como Antioquia, Risaralda, Cundinamarca, Meta o Valle del Cauca, presentarían los niveles más elevados de todo el país. La diferencia es tan grande que incluso el sur del país llega a presentar una brecha del $46 \%$ con respecto de la media de ingresos mensuales nacional, y del $47,4 \%$ con respecto de la región más próspera de Colombia. Algo similar sucedería con la línea de pobreza, que si ya de por sí es importante en el promedio nacional, habida cuenta de que casi uno de cada tres colombianos se encuentra en situación de pobreza y vulnerabilidad, en el caso de las regiones del sur, el nororiente y también el norte del país tal circunstancia termina por incrementarse en más de un $50 \%$.

Finalmente, con respecto al índice de calidad de vida ${ }^{7}$, cabe señalar que el $42,6 \%$ de los colombianos se percibiría a sí mismo en condiciones de pobreza, de modo que tal indicador, nuevamente, se incrementaría en más de un $40 \%$ en las regiones del nororiente, el sur y el norte del país.

No obstante, la excepcionalidad más relevante se encontraría en el coeficiente de Gini. Si bien este indicador suele utilizarse para entender y explicar dinámicas de violencia delincuencial, sobre todo en América Latina, lo cierto es que, para el caso colombiano, no se corresponde con los escenarios de mayor violencia. Sin embargo, las tres regiones con mayor nivel de violencia estructural y también con mayores dinámicas de violencia directa son las que, a su vez, presentan menores niveles de desigualdad. Es decir, son las dos regiones económicamente más prósperas — el centro y el occidente del país - las que terminan siendo más desiguales y menos violentas. Una cuestión en cierto modo paradigmática, que invita a realizar investigaciones de mayor profun-

6. El PIB per cápita por unidad de gasto familiar (mensual) es el indicador que mide los ingresos según el DPS.

7. El índice de calidad de vida se encarga de valorar el vínculo de las variables relacionadas con el acceso de la población a servicios públicos básicos, la vivienda, la composición familiar y los niveles de educación. 
Tabla 8. Percepción de las alcaldías sobre las posibilidades municipales frente a los retos del posconflicto, 2014-2015

\begin{tabular}{lcc}
\hline & $\begin{array}{c}\text { Evolución de las capacidades } \\
\text { institucionales }\end{array}$ & $\begin{array}{c}\text { Expectativas posconflicto. } \\
\text { Condiciones socioeconómicas }\end{array}$ \\
\hline Norte & 6,01 & 5,45 \\
Occidente & 6,62 & 5,94 \\
Central & 6,50 & 5,05 \\
Oriente* $^{*}$ & 5,53 & 4,12 \\
Sur & $(3,83)$ & $(2,65)$ \\
\hline
\end{tabular}

* Entre paréntesis, los datos para Arauca y Norte de Santander.

Fuente: elaboración propia.

didad sobre el factor de la desigualdad y su relación con la violencia directa dentro de un conflicto tan intrincado como es el colombiano.

Es, quizá, por todo lo anterior que cabe entender el posconflicto armado como un horizonte que debe suponer, a efectos de mitigar la violencia, una suerte de políticas públicas que deben trascender estrictamente de la seguridad y, por ende, de la noción de paz negativa, tan limitada como insuficiente. Con independencia de las políticas que deban orientarse en el país, particularmente sobre aquellos dos escenarios que parecen concitar mayores niveles de continuidad en el activismo guerrillero - como son el sur y el nororiente-, resulta imprescindible intervenir sobre el narcotráfico y los cultivos cocaleros de una manera mucho más integral y preventiva de lo realizado hasta el momento. Ello porque las políticas reactivas, de aspersión y erradicación llevadas a cabo hasta ahora se han mostrado por completo infructuosas y no terminan por servir de alternativa a la ingente violencia estructural que se presenta de manera ubicua en buena parte del país, y especialmente focalizada y severa en las regiones en las que la violencia directa presenta una mayor intensidad.

Quizá por todo lo anterior, unido a una tendencia creciente de cultivos y a una violencia derivada del conflicto y enquistada en la periferia, se puede entender el nivel de escepticismo que muestran los alcaldes y las alcaldesas cuando son preguntados sobre las capacidades institucionales o las expectativas útiles para gestionar un proceso de posconflicto armado, tal y como se puede apreciar en la tabla 8. Nuevamente, las dos regiones que presentan peores registros son, precisamente, la del sur y la del nororiente del país.

\section{Conclusiones}

La paz negativa, entendida como ausencia de guerra, se trata de un enfoque minimalista, reduccionista, que en Colombia ha perdurado durante décadas, si bien se intensificó especialmente durante los años de la política de seguridad democrática puesta en marcha por Álvaro Uribe. La llegada a la presidencia por parte de Juan Manuel Santos, en buena medida, tal vez, por la incapacidad 
de poder finalizar el conflicto armado interno estrictamente, y únicamente, desde una dimensión militar, abrió la posibilidad de entablar un diálogo que, iniciado a finales de 2012 con las FARC (y actualmente en vías exploratorias con el ELN), busca la desactivación del conflicto a través de intercambios cooperativos con las guerrillas.

Colombia ha entrado en una nueva dinámica, sobre todo tras la firma del acuerdo con las FARC el pasado 26 de septiembre de 2016. Un horizonte cada vez más próximo, que debe trascender de la infructuosa dimensión militar de finalizar el conflicto para transitar hacia una forma más política y socioeconómica de entender la construcción de paz. Un reto especialmente relevante en las áreas más periféricas y fronterizas del país, donde concurre una mayor presencia de cultivos ilícitos y una menor presión de la fuerza pública, así como unos niveles mayores de violencia estructural.

Esta última cuestión es imprescindible. Las regiones del norte, del sur y del nororiente presentan ingentes niveles de exclusión y rezago en cuanto a los principales indicadores socioeconómicos, lo cual coincide con las percepciones más pesimistas de las alcaldías a efectos de albergar esperanzas en el proceso de construcción de paz y superación de la violencia. Esto invitaría a pensar en la necesidad de articular toda una respuesta integral que se orientara a generar recursos, transferir competencias, incentivar tejido empresarial y deslocalizar producción más allá de los enclaves centrales del país. Expresado de otro modo, mientras el desempleo, la pobreza o la falta de presencia del Estado continúen irresolutas, las posibilidades de articular una paz positiva, que afronte las condiciones estructurales que sirven de soporte a la violencia, quedan desdibujadas.

Dada la mayor complejidad que presenta la paz territorial, entendida como ese proceso real, diferencial, articulado y adaptado a las contingencias territoriales de índole local, pareciera que hasta el momento el país se encuentra lejos de su materialización en buena parte de los escenarios planteados. Esto es así porque el énfasis discursivo del Gobierno sobre esta noción de paz adaptada a la localidad no parece corresponderse con las percepciones, las posibilidades ni los instrumentos desde lo que disponen las alcaldías para hacer frente a lo que, en inicio, representa un proceso de posconflicto armado y, de manera más compleja, un escenario de construcción de paz efectiva y duradera.

Por desgracia, la experiencia paramilitar da buena cuenta de lo anterior. Tras la disolución de 2005, por la que las Autodefensas Unidas de Colombia dejaron inicialmente las armas, con lo que se desmovilizó a más de 33.000 combatientes en apenas tres años, casi un 25\% de los mismos integrantes volvieron a la criminalidad. Como un antiguo jefe paramilitar nos confesaba en algún momento:

En nuestro caso, nosotros pasamos por la cárcel como dirigentes de la estructura. Sin embargo, por fuera, desamparados, quedaron 30.000 hombres con más puntería que cultura y, por ello, muchos de ellos terminarían conformando las Bacrim. Hay que hacer, por tanto, una importante función de seguimiento que garantice, a todos, la reinserción plena en la sociedad. 
De igual forma, los ejemplos centroamericanos dan buena cuenta de la importancia del orden local, en este caso de suerte de paz territorial. Una paz que se construye, necesariamente, desde antes incluso que la firma del acuerdo y que debe trabajar desde dinámicas bottom-up, en términos reales, participativos y horizontales. Empero, hasta el momento y habida cuenta de lo recogido en todo este trabajo, la paz territorial parece más una declaración formal de intenciones que una posibilidad efectiva de una nueva política pública que priorice el nivel local como escenario de construcción de paz.

\section{Referencias bibliográficas}

Aguilera, Mario (2010). Las FARC: La guerrilla campesina 1949-2010. Bogotá: Arfo. ÁvilA, Ariel y VALENCIA, León (2016). Los retos del posconflicto: Justicia, seguridad y mercados ilegales. Bogotá: Ediciones B.

AzAR, Edward (1990). The Management of Protracted Social Conflict: Theory and Cases. Aldershot: Dartmouth.

BARRETO, Miguel (2016). Laboratorios de paz en territorios de violencias. Bogotá: Universidad Jorge Tadeo Lozano.

Bejarano, Ana y Pizarro, Eduardo (2001). "The Coming Anarchy: The Partial Collapse of the State and the Emergence of Aspiring State Makers in Colombia». Workshop at the Punk Center of Internacional Relations. Toronto: Universidad de Toronto.

BETANCOURT, Darío (1991). «Los cinco focos de la mafia colombiana (1968-1988): Elementos para una historia». Folios, 2, 13-30.

BLAIR, Elsa (1993). Las fuerzas armadas: Una mirada civil. Bogotá: CINEP.

Boulding, Kenneth (1962). Conflict and Defense: A General Theory. Nueva York: Harper and Row.

- (1977). «Twelve Friendly Quarrels with Johan Galtung». Journal of Peace Research, 14, 75-86. $<$ https://doi.org/10.1177/002234337701400105>

Calvo, Fabiola (1986). Ejército Popular de Liberación: Una historia armada. Bogotá: VOSA.

CÁRDENAS, Juan David (2015). «Los medios de comunicación como actores (des)legitimadores: Algunas reflexiones acerca del rol de los medios de comunicación sobre la construcción de la opinión pública en torno al proceso de paz de La Habana». Análisis Político, 28 (85), 38-56. $<$ https://doi.org/10.15446/anpol.v28n85.56245>

CODHES (2011). «De la seguridad a la prosperidad democrática en medio del conflicto". Documentos CODHES, 23, 143 p.

CurLe, Adam (1976). «Peace Studies». The Year Book of World Affairs, 30, 5-13.

Dedring, Juergen (1981). «Toward Appropriate Peace Research». Peace and Change, 3, 1-17. <https://doi.org/10.1111/j.1468-0130.1981.tb00435.x>

ECHANDÍA, Camilo (2006). Dos décadas de escalamiento del conflicto armado en Colombia (1986-2006). Bogotá: Universidad Externado.

EIDE, Asbjørn (1974). «Méthodes et problemes de la recherché sur la paix: Le choix de valeurs». Revue International des Sciences Sociales, 26, 129-144. 
FALS BORDA, Orlando (1996). Región e Historia: Elementos sobre ordenamiento territorial y equilibrio regional en Colombia. Bogotá: Universidad Nacional de Colombia.

FALS BORDA, Orlando; GuZMÁN, Germán y UMAÑA, Eduardo (1962). La violencia en Colombia. Bogotá: Tercer Mundo Editores.

FUndACIÓN SEGURIDAD Y DEMOCRACIA (2008). «El debilitamiento de los grupos irregulares en Colombia, 2002-2008». Revista Coyuntura de Seguridad, 23, 5-11.

GalTung, Johan (2003). Trascender y transformar: Una introducción al trabajo de conflictos. México DF: Transcend-Quimera.

GilhodÈs, Pierre (1972). Las luchas agrarias en Colombia. Bogotá: La Carreta.

Hardin, Garret (1983). «Is Violence Natural?». Journal of Religion and Science, 18 (4) 405-413. <https://doi.org/10.1111/j.1467-9744.1983.tb00524.x>

HARTO DE VERA, Fernando (2004). Investigación para la paz y resolución de conflictos. Valencia: Tirant Lo Blanch.

LARA, Patricia (2014). Siembra vientos y recogerás tempestades. Bogotá: Planeta.

LEAL, Fernando (1994). El oficio de la guerra: La seguridad nacional en Colombia. Bogotá: Tercer Mundo Editores.

López DE LA Roche, Fabio (2015). «El gobierno de Juan Manuel Santos 2010-2015: Cambios en el régimen comunicativo, protesta social y proceso de paz con las FARC». Análisis Político, 28 (85), 3-37. <https://doi.org/10.15446/anpol.v28n85.56244>

MASSÉ, Frédéric y CAMARGO, Johanna (2012). Actores armados ilegales y sector extractivo en Colombia. Bogotá: CITPax.

Medina, Carlos (1990). Autodefensas, paramilitares y narcotráfico en Colombia. Origen, desarrollo y consolidación: El caso "Puerto Boyacá». Bogotá: Documentos Periodísticos.

- (1996). ELN: Una historia contada a dos voces. Bogotá: Rodríguez Quito Editores.

- (2011). Las FARC-EP y ELN: Una historia comparada. Bogotá: Universidad Nacional de Colombia.

Miall, Hugh; Mitchell, Chris; Rambsbotham, Oliver y Woodhouse, Tom (2015). The Contemporary Conflict Resolution Reader. Cambridge: Polity Press.

Ministerio de Defensa (2010). Logros de la Política de Consolidación de la Seguridad Democrática. Bogotá.

Morales, Jairo (2015). ¿Qué es el post-conflicto? Bogotá: Ediciones B.

MuÑOZ, Francisco (2000). La paz imperfecta. Granada: Universidad de Granada.

ODHDIH (s.f.). Sintesis de la violencia y la confrontación armada en Colombia, 19982012 y 2015. Bogotá: Presidencia de la República.

OQuist, Paul (1978). Violencia, conflicto y política en Colombia. Bogotá: Instituto de Estudios Colombianos.

OTERO, Diego (2007). Las cifras del conflicto armado en Colombia. Bogotá: INDEPAZ.

- (2010). El papel de los Estados Unidos en el conflicto armado colombiano. Bogotá: Aurora.

PÉCAUT, Daniel (2008). Las FARC, ¿una guerrilla sin fin o sin fines? Bogotá: Norma.

PNUD (2003). Informe Nacional de Desarrollo Humano para Colombia: El conflicto, callejón con salida. Nueva York: Naciones Unidas.

RAMírEZ, William (1990). Estado, violencia y democracia. Bogotá: Universidad Nacional de Colombia.

Rapoport, Anatol (1960). Fights, Games and Debates. Ann Arbor: University of Michigan Press. 
REYES, Alejandro (1988). «Conflictos agrarios y luchas armadas en la Colombia contemporánea: Una visión geográfica». Análisis Politico, 5, 7-29.

- (2009). Guerreros y campesinos: El despojo de la tierra en Colombia. Bogotá: Norma.

Ríos, Jerónimo (2016). "La narcotización del activismo guerrillero de las FARC y el ELN, 1998-2012». Revista UNISCI, 41, 205-233. <https://doi.org/10.5209/rev_RUNI.2016.n41.52680>

- (2017). Breve historia del conflicto armado en Colombia. Madrid: La Catarata.

RoJAS, Diana (2015). El Plan Colombia: La intervención de Estados Unidos en el conflicto armado colombiano (1998-2012). Bogotá: Debate.

RÖLING, Bert (1984). "Investigación sobre la paz». En: VV.AA. Los científicos de la carrera armamentista y el desarme. Barcelona: Serbal-UNESCO.

Romero, Mauricio (2011). La economía de los paramilitares. Bogotá: Debate.

RONDEROS, Teresa (2014). Guerras recicladas: Una historia periodística del paramilitarismo en Colombia. Bogotá: Aguilar.

Rotberg, Robert (2004). When States Fail: Causes and Consequences. Princeton: Princeton University Press.

Rummel, Rudolph (1998). Statistics of Democide: Genocide and Mass Murder from 1900. Berlín: LIT Verlag.

SALAS, Luis (2015). "Lógicas territoriales y relaciones de poder en el espacio de los actores armados: Un aporte desde la geografía política al estudio de la violencia y el conflicto armado en Colombia, 1990-2012». Cuadernos de Geografía, 24, (1), 157-172.

SÁNCHEZ, Gonzalo (1987). Colombia: violencia y democracia: Comisión de Estudios para la Violencia. Bogotá: La Carreta, 2009.

SÁnCHeZ, Gonzalo y MEERTENS, Donny (1983). Bandoleros, gamonales y campesinos. Bogotá: El Áncora.

ScHmid, Herman (1968). "Politics and Peace Research». Journal of Peace Research, 5, 217-232.

Torres, María (2011). Estado y coca en la frontera colombiana: El caso de Putumayo. Bogotá: CINEP.

UNODC (2013). Colombia: Monitoreo de cultivos de coca 2012. Viena: Naciones Unidas.

- (2016). Colombia: Monitoreo de cultivos de coca 2015. Viena: Naciones Unidas.

VÁsQUEZ, Teófilo; VARGAS, Andrés y ReSTREPO, Jorge (2011). Una vieja guerra en un nuevo contexto: Conflicto y territorio en el sur de Colombia. Bogotá: CINEP. 